\title{
Acute gastric dilatation due to binge eating may be fatal
}

\author{
Oguzhan Dincel, Mustafa Goksu \\ Department of General Surgery, Adiyaman University, Faculty of Medicine, Adiyaman, Turkey
}

\begin{abstract}
Acute gastric dilatation was first described by Duplay in 1833. Since the stomach has rich blood circulation, necrosis and perforation due to dilatation are rarely seen. Clinically, more than $90 \%$ of cases have complaints of vomiting. The most useful method to determine diagnosis and etiology is computerized tomography (CT). Medical treatment is suitable for cases that have no necrosis or peritonitis findings. Delay in surgical treatment increases the risk of mortality. The present case is that of a diagnosis of gastric perforation due to acute gastric dilatation in a 24-year-old female patient.
\end{abstract}

Keywords: Binge eating; gastric dilatation; intravascular disseminated coagulation; mortality.

G astric necrosis and perforation after gastric dilatation are rare because the stomach has a rich blood supply, but occurrence can be life threatening. Pathogenesis is still controversial. The most frequent complaints are abdominal pain and vomiting, and there is usually also abdominal bloating. Emergency surgery is required for patients with gastric necrosis and perforation [1-3]. The present case reports on gastric decompression and closure of perforation in a patient with acute gastric dilatation due to overeating.

\section{CASE REPORT}

A 24-year-old female was admitted to the emergency room (ER) with sudden onset of abdominal pain, nausea and vomiting after eating a heavy meal 6 hours previously. Patient reported eating an excessive amount of grapes and pomegranates hours prior. Her pain was sharp, severe, and worst in the epigastrium.

Physical examination revealed a thin, uncomfortable-appearing young woman. Patient exhibited abdominal swelling, rigidity, and diffuse tenderness with peritonitis on palpation. All other examination findings were non-contributory. When the patient presented to the ER, vital signs were as follows: blood pressure (BP) 125/80 $\mathrm{mmHg}$, pulse 75 beats/minute, respiratory rate 16 breaths/minute, and a body temperature of $36.5^{\circ} \mathrm{C}$. Amount of urine from urinary catheter placed in bladder was approximately zero. Nasogastric tube inserted into

Received: November 26, 2015 Accepted: February 07, 2016 Online: April 04, 2016

Correspondence: Dr. Oguzhan DINCEL. Adiyaman Universitesi Tip Fakultesi, Genel Cerrahi Anabilim Dali, Adiyaman, Turkey.

Tel: +90 4167816131 e-mail: droguzhandincel@yahoo.com

(c) Copyright 2017 by Istanbul Northern Anatolian Association of Public Hospitals - Available online at www.kuzeyklinikleri.com 
the stomach was not effective because it was full of undigested stomach contents. Chemistries and hemogram were normal. Abdominal computed tomography (CT) showed a grossly distended stomach occupying the entire abdominal cavity.

Coronal image of abdominal CT scan revealed distended stomach containing air and radiodense material, later determined to be grapes and pomegranates (Figure 1). Sagittal image of abdominal CT showed that stomach over-distended with food content led to displacement of intra-abdominal structures (Figure 2).

During systemic examination, patient was checked for cyanotic condition and no pulse could be detected in either of lower extremities. Moreover, pulse rose to tachycardic rate of $125-135$ beats/minute, respiratory rate was 30-35 breaths/minute and BP was $70 / 45 \mathrm{mmHg} 2$ hours after first checked. The patient was intensively resuscitated preoperatively with crystalloid intravenous (IV) fluids and

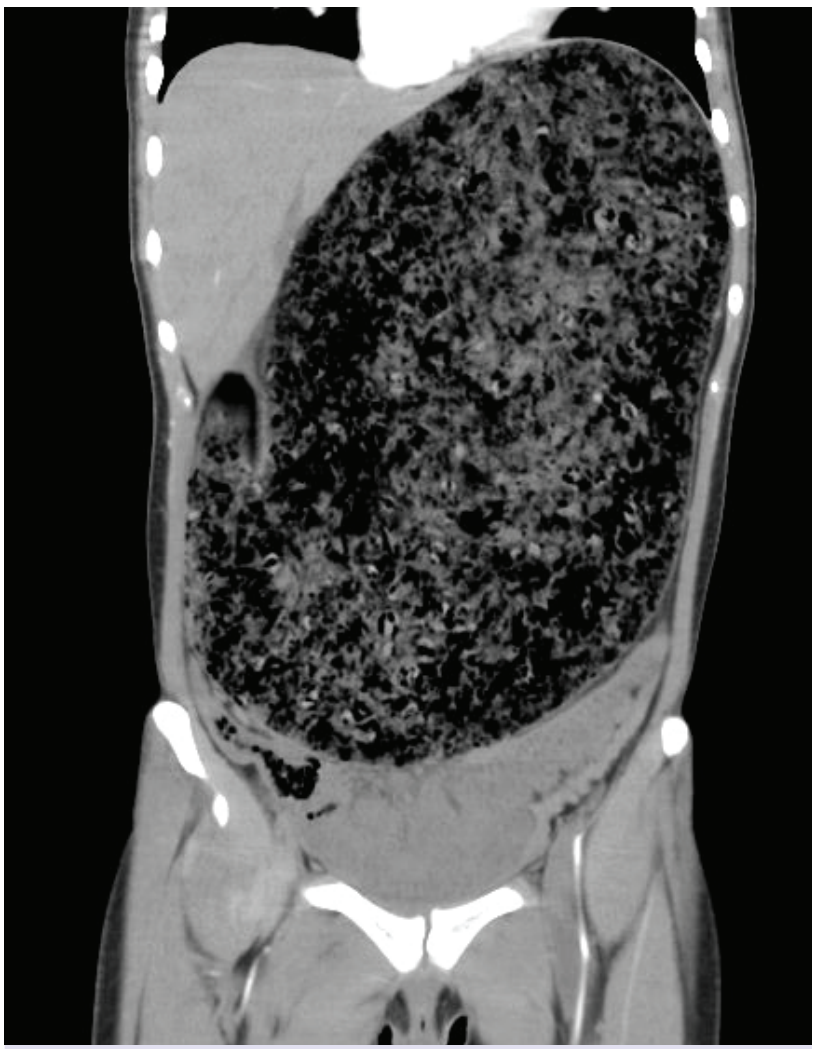

FIGURE 1. Distended stomach containing air and radiodense material. plasma expander. Shock occurred and patient's breathing worsened (oxygen saturation of $65 \%$ on air). An emergency operation was performed.

The patient underwent laparotomy (epigastric midline incision) that revealed massively distended stomach with perforation of gastric antrum. Stomach was filled with nearly 5 liters of partially and minimally digested food, including whole grapes and pomegranate matter. Following adequate decompression, stomach was moved from wound site, remaining contents were emptied, and perforation was closed (Figure 3). No discrete bezoar or mechanical gastric outlet obstruction was identified.

\section{DISCUSSION}

Duplay first defined acute gastric dilatation in 1833 [4]. Although there is no mechanical obstruction in acute gastric dilatation, stomach becomes increasingly hypotonic and over-stretched [5]. A heavy

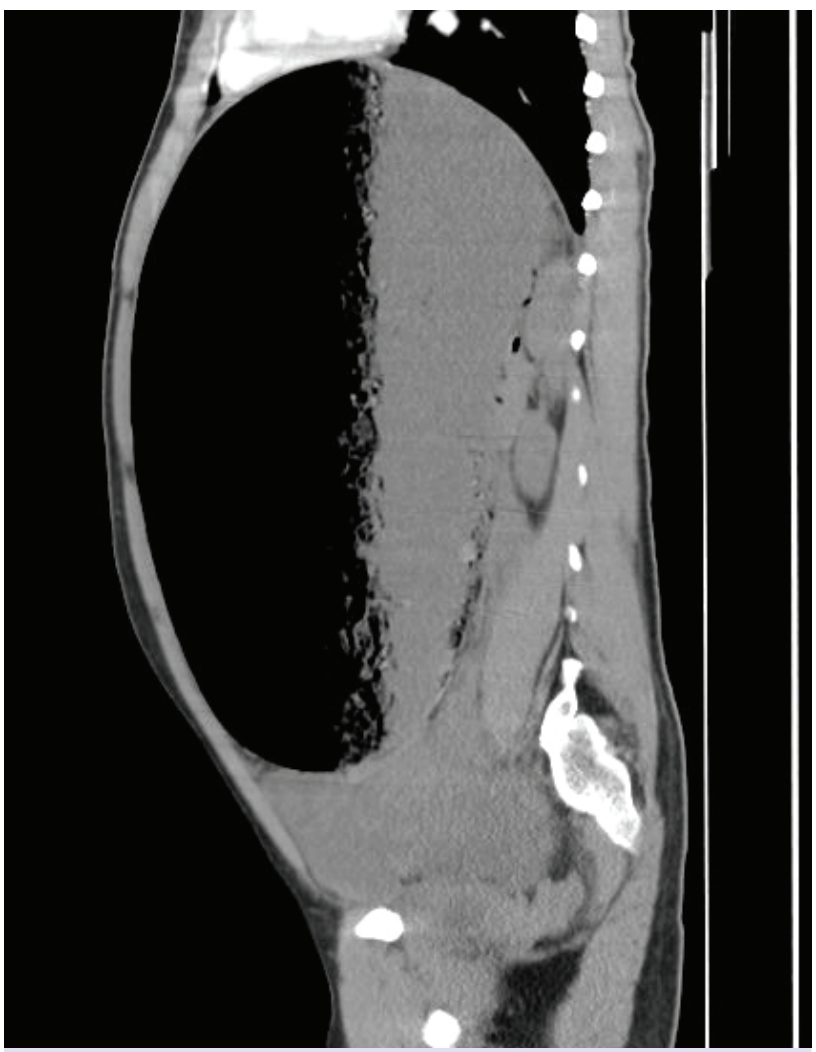

FIGURE2. Distended stomach containing food material. 


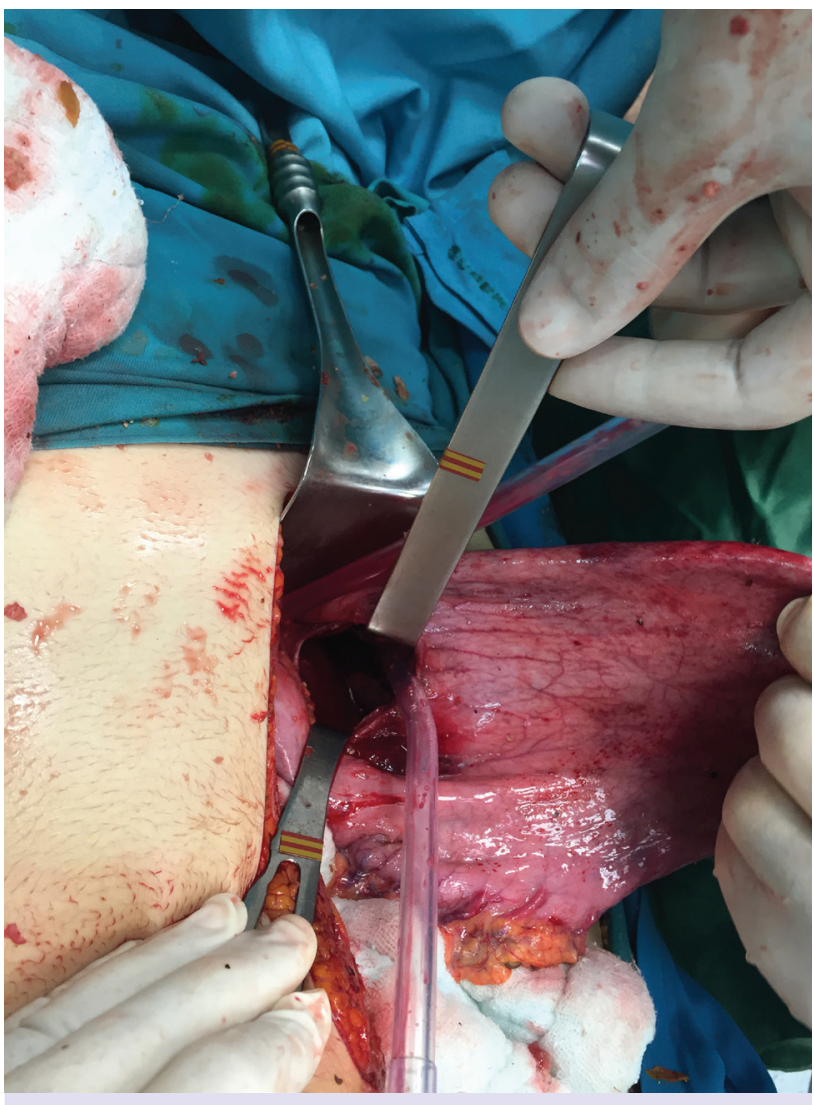

FIGURE 3. Perforation of gastric antrum.

meal, adhesive ileus, infection, parturition, central nervous system damage, and trauma are some causes of gastric dilatation [6].

Acute gastric dilatation due to overeating is more common in females with underlying eating disorders, such as anorexia nervosa [7]. In the present case, the patient was female and had eaten an excessive quantity of grapes and pomegranates.

Because of its extensive vascular network, the stomach is usually protected from ischemia, so necrosis and perforation of the stomach are rare. Due to the fact that excessive gastric dilatation increases intragastric pressure, intramural blood circulation can be reduced, possibly leading to gastric necrosis or perforation [8]. In the present case, perforation was observed in the gastric antrum.

Rapid gastric decompression and appropriate fluid resuscitation reduce the pressure inside the stomach, thus preventing necrosis and perforation of the stomach. For that reason, these methods should be employed as early as possible [9]. In the present case, there was not effective gastric drainage and due to an increase in intragastric pressure, a perforation occurred.

Clinically, more than $90 \%$ of cases present with vomiting. Epigastric fullness, abdominal distention and pain may also be present $[10,11]$. The present patient experienced abdominal pain, nausea, and vomiting.

Abdominal distension, epigastric sensitivity, defence and rebound tenderness can be detected on physical examination. Abdominal x-ray may reveal a large air-fluid level that belongs to the fundus of the stomach, and subdiaphragmatic free air in cases with perforation. The most useful imaging modality is abdominal CT, which is not only useful in diagnosis but also in revealing the etiology [12].

The excessive increase in stomach contents through acute gastric dilatation compresses the gastric wall and leads to necrosis and perforation of the stomach. It also leads to compression on the inferior vena cava, and causes hypotension due to the decrease in venous return. Acute gastric dilatation can be controlled by nasogastric decompression and appropriate fluid-electrolyte therapy. Upper gastrointestinal endoscopy can help decompression. Medical treatment is suitable in cases without signs of peritonitis on examination $[1,4]$.

Advanced cases with necrosis and perforation require surgical intervention [1]. Mortality rates after delayed surgery in patients with necrosis and perforation are reported to be as high as $80 \%$ [13]. Surgical treatment options include surgical decompression, partial gastrectomy, total gastrectomy and esophago-jejunostomy, total gastrectomy with cervical esophagostomy, and feeding jejunostomy [1, $3,4,11]$. In the present case, perforation at the antrum of the stomach was detected during operation. Therefore, decompression and simple closure were performed. However, the patient died on the first postoperative day due to sepsis and disseminated intravascular coagulopathy.

Acute gastric dilatation is a condition to be considered in patients with eating disorders. The sus- 
pected diagnosis and early management can avoid complications and decrease mortality substantially.

Conflict of Interest: No conflict of interest was declared by the authors.

Financial Disclosure: The authors declared that this study has received no financial support.

Authorship contributions: Concept - O.D., M.G.; Design O.D., M.G.; Data Collection - O.D., M.G.; Analysis - O.D., M.G.; Literature search - O.D., M.G.; Writing - O.D.

\section{REFERENCES}

1. Turan M, Sen M, Canbay E, Karadayi K, Yildiz E. Gastric necrosis and perforation caused by acute gastric dilatation: report of a case. Surg Today 2003;33:302-4.

2. Bortul M, Scaramucci M, Tonello C, Spivach A, Liguori G. Gastric wall necrosis from organo-axial volvulus as a late complication of laparoscopic gastric banding. Obes Surg 2004;14:285-7.

3. Lim JE, Duke GL, Eachempati SR. Superior mesenteric artery syndrome presenting with acute massive gastric dilatation, gastric wall pneumatosis, and portal venous gas. Surgery 2003;134:840-3.

4. Todd SR, Marshall GT, Tyroch AH. Acute gastric dilatation revisited. Am Surg 2000;66:709-10.

5. Hiraga M, Ono F, Omura N, Sato M, Yamamura A. A case of gastric necrosis and perforation due to overeating-induced gastric dilatation: A case report. J Jpn Surg Assoc 2012;73:1933-37.

6. Kaneda T, Miyazawa H, Kobayashi T, Shimizu N, Katayama M, Sato T. A case of acute gastric dilatation occurring after bulimia while on a diet. J Tokyo Med Univ 1996;54:66-8.

7. Natsume S, Terasaki M, Goto Y, Kurumiya Y, Shingu Y. A case of gastric necrosis. J Abdom Emerg Med 2003;23:1075-8.

8. Usuda M, Koizumi M, Kouda H, Nakahara C, Ueki H, Shibazaki S. Gastric necrosis caused by acute gastric dilatation after an episode of bulimia-A case report. Jpn J Gastroenterol Surg 1998;31:2346-9.

9. Nakao A, Isozaki H, Iwagaki H, Kanagawa T, Takakura N, Tanaka N. Gastric perforation caused by a bulimic attack in an anorexia nervosa patient: report of a case. Surg Today 2000;30:435-7.

10. Chaun H. Massive gastric dilatation of uncertain etiology. Can Med Assoc J 1969;100:346-8.

11. Holtkamp K, Mogharrebi R, Hanisch C, Schumpelick V, Herpertz-Dahlmann B. Gastric dilatation in a girl with former obesity and atypical anorexia nervosa. Int J Eat Disord 2002;32:3726.

12. Adson DE, Mitchell JE, Trenkner SW. The superior mesenteric artery syndrome and acute gastric dilatation in eating disorders: a report of two cases and a review of the literature. Int J Eat Disord 1997;21:103-14.

13. Koyazounda A, Le Baron JC, Abed N, Daussy D, Lafarie M, Pinsard M. Gastric necrosis caused by acute gastric dilatation. Total gastrectomy. Recovery. J Chir (Paris) 1985;122:403-7. 